Article

\title{
Efficient Economic and Resilience-Based Optimization for Disaster Recovery Management of Critical Infrastructures
}

\author{
Eng Tseng Lau ${ }^{1} * \mathbb{D}$, Kok Keong Chai ${ }^{1}$, Yue Chen ${ }^{1}$ and Jonathan Loo ${ }^{2}$ \\ 1 School of Electronic Engineering and Computer Sciences, Queen Mary University of London, \\ Mile End Road, London E1 4NS, UK; michael.chai@qmul.ac.uk (K.K.C.); yue.chen@qmul.ac.uk (Y.C.) \\ 2 School of Computing and Engineering, University of West London, St Mary's Road, London W5 5RF, UK; \\ jonathan.loo@uwl.ac.uk \\ * Correspondence: e.t.lau@qmul.ac.uk; Tel.: +44-1895-268-368
}

Received: 2 November 2018; Accepted: 30 November 2018; Published: 6 December 2018

check for updates

\begin{abstract}
The traditional grid operation is unfortunately lacking the resilience and responsiveness in reacting to contingency events due to the poor utilization of available resources in mitigating the shortfalls. Such an unaddressed issue may affect the grid stability and the ultimate grid blackout. Therefore, this paper models a grid optimization module consisting of a mid and low (microgrid) voltage level grid component of an urban grid network for a disaster recovery. The model minimizes the cost of generation required to meet the demand through the economic dispatch in combination with the unit commitment. Two optimization problems are formulated that resemble the grid operation: normal (grid-connected) and islanded. A constrained-based linear programming optimization problem is used to solve the formulated problems, where the dual-simplex algorithm is used as the linear solver. The model ensures sufficient demand to be met during the outages through the $\mathrm{N}-1$ contingency criterion for critical infrastructures. The simulation length is limited to $24 \mathrm{~h}$ and is solved using the MATLAB ${ }^{\circledR} \mathrm{R} 2017 \mathrm{~b}$ software. Three different cases are established to evaluated the modelled grid resilience during the grid-connected or the islanding of operations subject to adversed events. The simulated results provide the economical outage recovery that will maintain the grid resilience across the grid.
\end{abstract}

Keywords: distributed energy resources; energy management system; resilience; optimization; smart grids

\section{Introduction}

The traditional grid has been criticized due to the one way of communication flow (top-bottom) and therefore is unable to response quickly to emergency events. The targeted grid attacks, unplanned contingencies or natural disaster phenomenon may pose a significant risk of power grid failures, which may subsequently cause partial or complete electricity blackouts. Long-term electricity blackouts without restorations do not only cause huge disruptions within communities but also may significantly reduce the economic and social welfare. For this reason, the smart grid concept brings into the action in order to compensate such shortcomings. Through the integration of information and communication technology (ICT) in the smart grid, this allows the prompt bi-directional communication flow within the electrical grid [1,2]. Such mechanism speeds up the energy trading, response and recovery purpose as before the communication may have broken down completely (assuming standby generations are available to power up the communication system for a short time period) after a disaster outbreak. This prevents the need to rely on the irreversible top-bottom communication flow, which is slow and 
ineffective in responding to unplanned contingencies (as the communication system may have already failed before reaching to the bottom level).

A microgrid, on the other hand, is an integrated low-voltage (LV) power delivery system in the smart grid composed of loads, a distributed energy resource (DER) such as a standby generator (non-renewable energy source), a renewable energy source (RES) and an energy storage system (ESS) operating as a single controllable load connected to the main grid [1,3]. The microgrid has the ability to isolate its LV grid portion from the main grid (grid-connected) or the partial isolation (islanding) within the LV network, in the case of outage/contingency events [1].

There are two types of microgrid controls within the smart grid network: centralized and decentralized control. According to Vaccaro et al. [4] and Olivares et al. [5], a core microgrid controller in the centralized control provides appropriate control actions (set points) to the local controllers of different DER units and loads that optimizes the local energy production and the main grid. A decentralized control, on the other hand, allows the full autonomy for all local units, where units are 'smart' and can communicate with each other to form a larger topology. In present, both control mechanisms have their drawbacks. According to Vaccaro et al. [4] and Olivares et al. [5], a fully centralized control is infeasible to be deployed in geographically sparse areas, where extensive communication infrastructure and computations are required. On the other hand, a fully decentralized approach is not possible due to strong coupling points within units and localised information. Hence, the system will not be aware the overall system operation and the control actions from the microgrid controller. As asserted by Hatziargyriou et al. [6] and Erol-Kantarci et al. [1] and the main finding by Winkler et al. [7], the presence of generation in proximity of the demand is critical to enhance the overall power reliability and quality. As holistic information of the grid is required in responding to adverse events, a centralized microgrid control is considered in this paper.

The energy management system of microgrid is based on the localised controllable DER and loads with the combination of the Unit Commitment (UC) and Optimal Power Flow (OPF) models $[8,9]$. The main aim of the microgrid is to optimally allocate the DER that will minimize the monetary cost and increase the performance of grid operations. Elsied et al. [8] proposed an advanced real-time energy management system for the microgrid in order to achieve the optimal allocation of DER. The optimization model consisted of a generalized economic cost as the objective function with operational constraints and the problem was solved using the Binary Particle Swarm optimization (PSO) algorithm. Meanwhile, Shi et al. [10] proposed a centralized-based distributed energy management system where the microgrid central controller and the local controllers were joined to compute an optimal schedule. The system applied complex optimal power flow as the problem formulation. Parisio et al. [11] presented a Model Predictive Control (MPC) with Mixed Integer Linear Programming (MILP) framework which included the UC, economic dispatch and ESS for grid interaction and curtailment schedule in an experimental microgrid located in Athens. Hossienzadeh and Salmasi [12] proposed a robust optimal power management system for an isolated AC/DC microgrid, where the system performance was assessed through MILP-based minimization of the cost function with operational constraints. The system also considered the uncertainties in the power output and forecast generation errors, and two-level-based control mechanism of charging/discharging storage batteries. A smart energy management system to optimize the operation of microgrid was presented by Chen et al. [13] where the optimization problem of ESS, economic load dispatch and the optimization of DER was simplified into a single-objective problem. Yang et al. [14] proposed a novel stochastic optimal control strategy focusing in independent microgrids based on the integration of ESSs and traditional synchronous generators. The approach was based on an improved design of linear quadratic Gaussian controller and the consideration of the ESS's current state of charge (SOC) that prevented the batteries' overcharging and overdischarging problems, thus adaptively regulating the power output to minimize the frequency fluctuations. Wang et al. [15] proposed a hierarchical three-level multi-agent system (distribution network, microgrid and distributed generation level) and decentralized technique for optimal coordination and operation of several AC/DC hybrid microgrids. 
At the microgrid level, the MILP was performed in an individual microgrid and a consensus algorithm was further utilized to optimally coordinate multiple microgrids. Interestingly, a fuzzy logic-based artificial neural network power management system was proposed by Chettibi et al. [16] to minimize the energy purchased from the electrical grid through maximum untilization of RES and ESS subject to variable conditions such as climate, demand and disturbance in electrical grids.

Overall, the intensive concentration in optimal allocation of DER is the main target that reduces the microgrid operation cost, and increases the stability and performance. However, less attention has been paid to microgrid approaches in the grid resilience subject to adverse events, as well as the quantification of grid resilience. The enhanced performance and economical operation cost may not reflect the desired grid resiliency. As indicated by Panteli and Pierluigi [17], the cost-efficient solution might indicate the extra investment of smart technologies, but the degree of robustness of the investment might be uncertain. Therefore, conceptualising the concept of resilience in power grids may help in balancing the monetary costs and resilience.

According to Panteli and Pierluigi [17], Cano-Andrade et al. [18], Bollinger [19], Khodaei [20] and Liu et al. [21], resilience is the ability of a power system to withstand/remain in a state during a failure in an efficient manner, and to quickly restore to the normal operating state. A generic resilience framework '4Rs' - robustness, redundancy, resourcefulness, and rapidity-was developed by the Multidisciplinary and National Center for Earthquake Engineering Research to be applicable for critical infrastructures [17]. Four resilience indices are introduced by Liu et al. [21], which are the number of line outages, loss of load probability (LOLP), expected demand that cannot be supplied, and the difficulty of grid recovery indexes. However, resilience assessments as introduced by Liu et al. [21] contradicted with the resilience computation by Cano-Andrade et al. [18], Bollinger [19], as the grid resilience in this case is determined by the extent in which the amount of energy demand within consumers are met when there is a disturbance in the grid.

Several resilience-based power system operations have been considered to address the disaster and contingency events. Romero et al. [22] applied stochastic programming to optimize the capacity enhancement strategies of the transmission and generation that increased the resilience of large-scale power systems against seismic risks (earthquakes). Winkler et al. [7] presented a new methodology combining the hurricane damage predictions and different grid topological assessments to characterise the impact of hurricanes towards the reliability of the power grid. The analysis concluded that the ring-meshed grid topology provided superior performance during hurricane hazards due to the presence of a centralized substation ring. Ouyang and Dueñas-Osorio [23] introduced the time-dependent resilience assessment of urban infrastructure systems. A resilience-oriented microgrid optimal scheduling model was proposed by Khodaei [20] that minimized the reduction in microgrid load by efficiently scheduling the available resources at a point of supply interruption from the main grid. A holistic framework for optimal microgrid operation to guarantee uninterrupted power supply to critical loads was proposed by Detroja [24]. The framework consisted of three optimization modules to perform the load scheduling, UC and power balancing. Bahramirad [25] presented the economic and emergency operation of ESS in a microgrid, by introducing outages within generation units and transmission lines. Erol-Kantarci et al. [1] focused on clustering methods that multiple smart microgrids (SMGs) would operate collaboratively to form a smart microgrid network (SMGN) of a ring topology. The main aims are to accelerate the deployment of low-carbon energy resources and to ensure the survivability of the SMGN through resource sharing within smart grid networks. The approach, however, neglected the cost function and the grid resilience. The full utilization of low carbon energy resources may not guarantee the low operational cost and the grid resilience.

However, the lack of time-dependent resilience quantification and assessment impede the justification of microgrid penetrations in enhancing grid resilience. Still, the resilience concept was only briefly introduced in the context. Ouyang and Dueñas-Osorio [23] asserted that the time-dependent resilience analysis enabled the evolvement in the power grid, the process improvements and the possibility of grid underperformance issues. Additionally, resilience assessments were particularly 
being focused within large scale applications of generation, transmission and the bulk grid in the sparsely populated area.

In this regard, this paper aims to model a holistic grid distribution network composing the MV (mid-voltage) and LV (low voltage) level of an urban grid. The model further mitigates the impact of supply failure during disasters and emergency responses, especially in the critical infrastructures that are highly dependent on the availability of electricity. The contributions of the proposed method are as follows:

(i) The proposed model is a novel approach that creates a new methodology to specifically illustrate the time-dependent-based grid resilience during the grid recovery process for an outage event;

(ii) The modelled system enables utility planners or engineers to foresee the grid structure that is vulnerable to outages (partial or complete), as well as generation intermittencies and failures;

(iii) The simulated model allows the most economical approach of meeting demand shortage towards critical infrastructures while maintaining the grid resilience;

(iv) The possibility of an overloaded electrical grid can be prevented and thus defers the need for network reinforcements.

The paper is organised as follows: Section 2 describes the system model for the grid architecture. Section 3 describes the formulation problem and the proposed solution of the optimization problem, the modelling of ESS and the resilience calculation. Section 4 presents the model simulation settings prior the simulation. Section 5 demonstrates case studies of an urban grid for normal and outage operation. Section 6 provides conclusions.

\section{System Model}

A common microgrid configuration is presented in Figure 1. Each node (Node 1, Node 2,..., Node $M+N$ ) includes the component of MV and LV which includes the generator (in the MV level), electrical loads for the end consumers, and small scale diesel-powered standby generators, ESS and RES that are connected via electrical interconnectors for the LV level. Both $M$ and $N$ denote the number of nodes for the MV and LV level correspondingly. The feeders transfer the power from generators to the end consumers [26]. The microgrid is connected to the MV grid through a transmission line. A circuit breaker (CB), $C B 1$, is installed that acts as the protection mechanism to connect or disconnect the MV grid from the high level grid. Similarly, $C B 2$ is used to protect the LV microgrid from the MV grid.

The grid can have two operation modes: normal and islanded state. In normal state, the microgrid is interacted with the MV grid. On the contrary, during an outage event, the microgrid can operate in an islanded mode by opening $C B 2$ in Figure 1 . This is to enable the continuous supply of energy within the LV microgrid without interruptions. The microgrid controller will send control actions to standby generator and ESS to provide energy to the microgrid. Similarly, the MV grid can still operate through on-site 'self-generation' by opening CB2. In addition, an islanded operation can also occur within a linked connection in the microgrid. For example, all the components in Node 3 of the microgrid can operate in islanded mode if $C B 5$ is opened. The remaining nodes operate normally and interact with the MV grid.

It is assumed in this case that the microgrid controller has the capability to perform switching by isolating the LV network from the grid entirely due to the sophisticated design and capability of CBs. This is because there is an electronic control applied at the gateway controller, which is relevant to the protection and reliability operation schemes during a contingency and when switching from high voltage generation to MV-LV generation. However, it is not the scope of this paper to study the switching, protection and dynamic operation performance. Additionally, during an outage event, we assumed that the electricity line connections within the islanded node (the connection between a DER and a critical infrastructure) would have robust design features where energy is delivered without suffering significant energy losses, regardless of the location. Henceforth, choices of the 
geographical location and the robust design features of electricity connections would not affect the microgrid operation in this case.

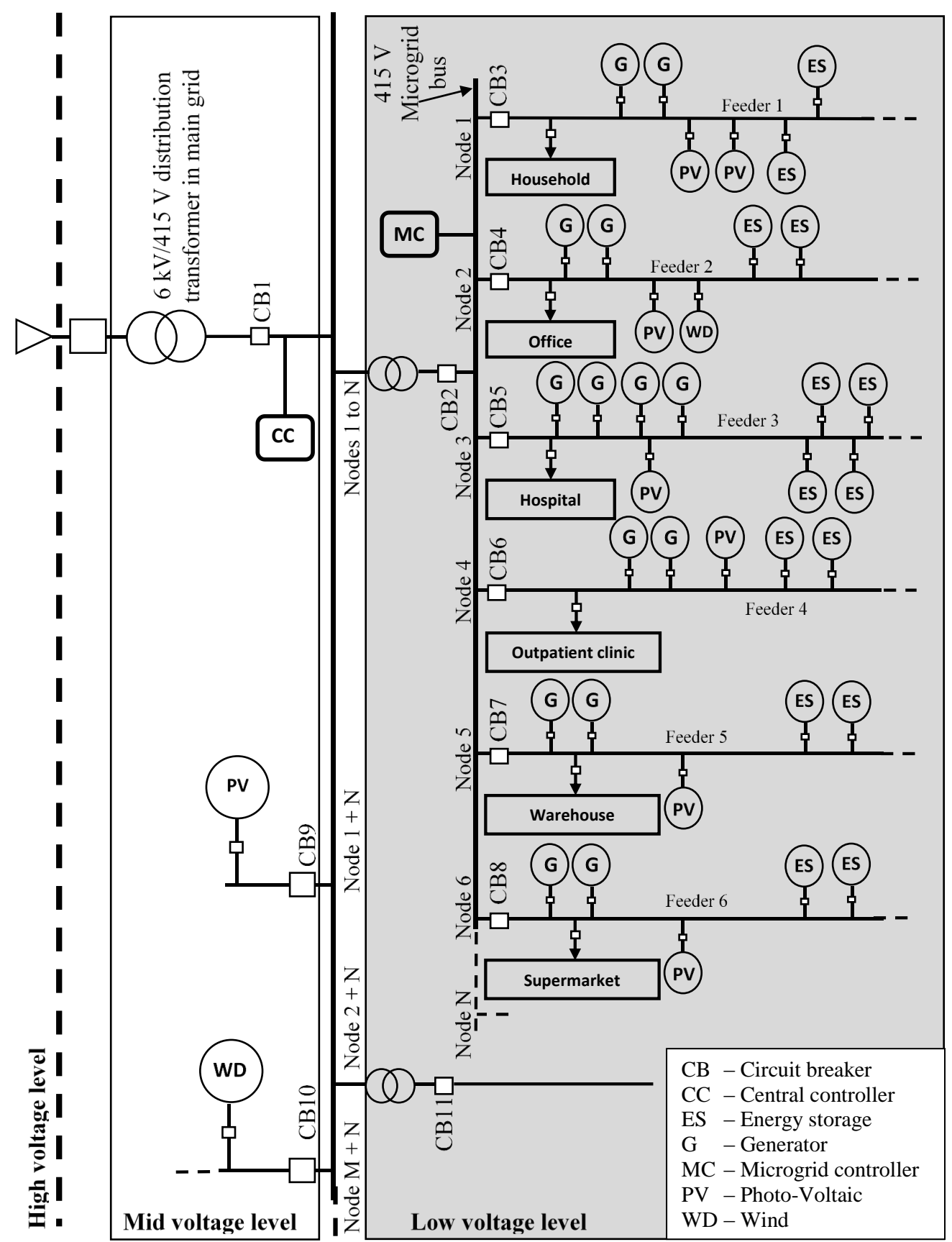

Figure 1. A microgrid configuration, adapted and modified from Liang and Zhuang [26].

The system model in this paper concentrates on the centralized perspective of the microgrid controller in optimizing the localised energy productions. This is achieved by performing an energy balancing optimization based on a cost minimization function of the DER such as standby generator, ESS and RES. Through the energy balancing optimization algorithm, the model automatically identifies the most economical approach of meeting the demand shortage within critical infrastructures while maintaining the grid resilience. 


\section{Problem Formulation}

As before with proceeding to the problem formulation of the grid optimization, a flow chart illustrating the integration process of the proposed grid optimization module that optimises the monetary costs and maintaining the grid resilience is demonstrated in Figure 2. The process begins with the determination of the current grid operation state. If no outage occurs, the grid operates in normal mode and the so-called 'economic-based' islanding operation is introduced to prevent the LV level grid from drawing the electricity from the main grid at times of high electricity price. This allows the optimal cost operation between the MV/LV level grid in responding to the market price. In contrast, if outage occurs, the portion of electrical grid with outage is isolated from the remaining grid portion and the specialized islanded grid optimization module is used to find the optimal operational cost of dispatching generating units in responding to sudden demand shortage due to an outage. For both grid operational modes, the performance of optimizations is evaluated through cost savings and resilience analysis.

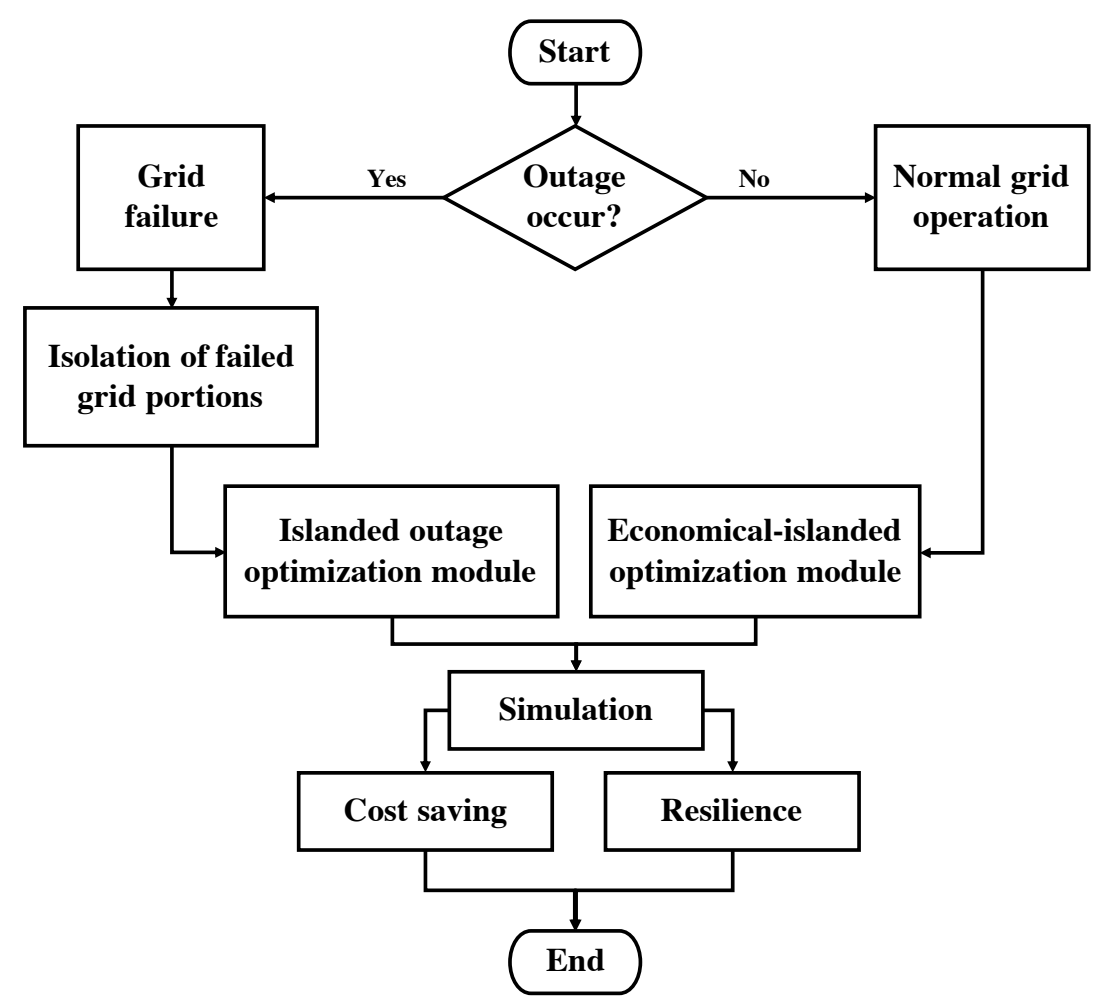

Figure 2. Flow chart describing the integration process of the proposed grid optimization module.

\subsection{Grid Optimization Problem}

The optimization problem is typically the economic dispatch in the combination of the UC problem. The economic dispatch optimizes the most economical way of dispatching generating units (except RES) while meeting the nationwide demand. Due to the nature of generation intermittency within RES, the generation output shortages within RES are balanced by available standby generators, mid-scale power plant and ESS. Hence, there is no associated optimization performed in RES in this case. Lau et al. [27] provide detailed fuel consumption and cost function in the economic dispatch problem. In contrast, the UC problem deals with coordinations of committable and non-committable state of standby generators, as well as the varied loads, based on hourly (h) intervals of scheduling slots.

The optimization problem formulations from Bahramirad [25], Zendehdel et al. [28], Hedman et al. [29], Khodaei [30], Howlader et al. [31] are followed. A single objective function optimization problem is proposed in this paper, with the combination of economic dispatch and UC problems. This is to reduce the complexity and prevent the arising of multi-objective optimization problems [32]. The objective 
function, as well as constraints, are formulated based on the linear programming (LP) problem. This is to prevent the arising of nonlinear problems. In fact, a constrained linear programming is a convex optimization problem that has an extreme point of the feasible set, and thus will have an optimal solution. Since the adaptive and better quality of decision is peculiarly critical in an emergency situation; therefore, LP optimization problem is highly recommended [33]. The model also presumes that the microgrid has more energy generation than loads for all nodes in the microgrid and no load curtailment for the node, or for the loads that are implemented in this case.

\subsection{Objective Function for Normal Operation}

The objective function for normal operation $\left(C_{N}\right)$ as formulated in Equation (1) is based on three important terms, where the first term is the operating cost of the dispatched units, the second term deals with ESS and the final term deals with the cost of the electricity purchased from the main grid (high level):

$$
\text { Minimize: } \quad C_{N}(t)=\sum_{g=1}^{N_{g}}\left[F_{g}\left(P_{G, g t}\right) I_{g t}+S U_{g}+S D_{g}\right]+\sum_{s=1}^{N_{s}}\left[C_{t} P_{S, s t}\right]+C_{t} P_{M, t} .
$$

The $g, s$ and $t$ index the corresponding generating units (diesel-powered standby generation units), ESS and the time period. $N_{g}$ and $N_{S}$ denote the standby generators, ESS and the time period. $P_{M, t}$ is the transmission line power. $P_{G, g t}$ is the energy dispatch of corresponding unit $g . S U_{g}$ is the start-up cost. $P_{S, s t}$ is the energy generated in $s$ at time $t . S D_{g}$ is the shutdown cost. $C_{t}$ is the electricity market price at the time where there exists electricity connection from the LV to MV level. $I_{g t}$ is the commitment state binary value of unit $g$.

Ever since the wholesale electricity market liberalization in early 1990s, the wholesale electricity market has become a very fundamental parameter to balance the acceptable costs for consumers' demand, security of supply and the fulfilment of environmental policy from the national to global level [34,35]. Weron [34] provided a very useful guideline on the electricity market trading process that involved typically a day-ahead auction market, where interested parties submit their bids and offers for the delivery of electricity based on their availability of generation capacity for the next day as before the trading market is closed. There are a variety of methods and ideas adopted for optimal electricity price forecasting. However, it is not the scope of this paper to forecast the day-ahead electricity price. Instead, for simplicity purposes, the electrical exchange data will be obtained from a public domain.

In Equation (1), the first term refers to the generation cost that is denoted by $F_{g}\left(P_{G, g t}\right)$. For thermal units, the generation cost is presented in a quadratic function and is simply approximated by a piecewise linear approximation. The generator's committable state is represented by the variable $I_{g t}$. $I_{g t}$ equals one when the unit is committable and zero when the unit is not committable. Hence, there will be a generation cost when the commitment state of the unit is one and, in contrast, zero generation cost when the unit is not committed. The $S U$ and $S D$ of thermal units are also modelled explicitly. The $S U$ variable is introduced as $u_{g t}$ and, in contrast, the $S D$ variable as $w_{g t}$. Both $u_{g t}$ and $w_{g t}$ are binary variables. When unit $g$ is switched on at current time $t$ but has been switched off until $t-1$, the $S U$ is imposed with $u_{g t}=1$. Otherwise, $u_{g t}=0$. Similarly, when $g$ is switched off at time $t$ but has been switched on until $t-1$, the $S D$ cost will be imposed with $w_{g t}=1$ and, otherwise, $w_{g t}=0$. The relationship of $u_{g t}$ and $w_{g t}$ with the UC variable $I_{g t}$ can be presented as:

$$
u_{g t}+w_{g t}=I_{g t}-I_{g(t-1)} \text {. }
$$

For the second term, there is ESS cost when the energy is drawn from the main grid (ESS is in charging mode) at times of low electricity market prices $C_{t}$. On the contrary, during the generation (discharging mode), zero generation cost is assumed to be imposed by ESS. 
For the final term, depending on the operation state, there will be electricity costs when there is an interaction between the present and the main grid. In contrast, there will be zero electricity costs when no energy is drawn from the main grid.

The objective function as formulated in Equation (1) optimizes $C_{N}$ at every time step $t$ (e.g., current time $\left.t=1,2, \ldots, N_{t}\right)$. The grid is further subject to the following operational constraints:

\subsubsection{The Power Balance Constraint}

The power balance constraint ensures that the total power generation from the LV and MV units would satisfy the overall load. It is defined as:

$$
\sum_{g=1}^{N_{g}} P_{G, g t}+P_{M, t}+\sum_{r=1}^{N_{r}} P_{R, r t}+\sum_{s=1}^{N_{s}} P_{S, s t}=P_{D, t}
$$

where $P_{D, t}$ is total aggregated energy across the grid, $r$ indexes RES and $P_{R, r t}$ is the energy generated from RES. $N_{g}, N_{r}$ and $N_{S}$ are the total number of non-renewable generators, RES and ESS, respectively.

\subsubsection{Unit Output Limit}

The generation units are imposed by minimum and maximum amount of generation capacities:

$$
P_{G, g}^{\min } I_{g t} \leq P_{G, g t} \leq P_{G, g}^{\max } I_{g t}
$$

where $P_{G, g}^{\min }$ is the minimum allowable capacity and $P_{G, g}^{\max }$ is the maximum allowable capacity of $P_{G, g t}$ at unit $g$.

\subsubsection{Ramp up and down Constraints}

The ramp up and down rate is formulated as:

$$
\begin{aligned}
& P_{G, g t}-P_{G, g(t-1)} \leq U R_{g}, \\
& P_{G, g(t-1)}-P_{G, g t} \leq D R_{g},
\end{aligned}
$$

where $U R_{g}$ is the ramp up rate limit and $D R_{g}$ is the ramp down rate limit. The imposed ramping constraint limits the amount of a generating unit within two successive $h$. That is to say, a generator is not allowed to reach a maximum capacity due to the nature of operations [27].

\subsubsection{Transmission Line Capacity Constraint}

The transmission line capacity limit between MV/LV level grid bus is formulated in Equation (7). Equation (8) formulates the distribution line limits within the LV level:

$$
\begin{aligned}
& \left|P_{M, t}\right| \leq P_{M}^{\max } \\
& \left|P_{G, g t}\right| \leq P_{G, g}^{\max } .
\end{aligned}
$$

\subsubsection{Spinning Reserve Requirement}

The spinning (or operating) reserve is the additional required generating capacity and is made available to the electrical system operator within a short time interval to meet demand in case of contingency events such as generation or transmission failures, or sudden peak demands. The spinning reserve allows the responsive and speedy recovery of energy shortages (e.g., within $10 \mathrm{~min}$ ) through fast acting generators that feed to the electrical grids. The spinning reserve requirement is formulated as: 


$$
\sum_{g=1}^{N_{g}} P_{G, g}^{\max } I_{g t}+P_{M}^{\max } \leq P_{D, t}+R_{t} .
$$

The $R_{t}$ is the reserve requirement at time $t$. In this case, the spinning reserve is online but unloaded, which will be triggered by the central or the microgrid controller to commit if required. $R_{t}$ in this case is calculated as the percentage of the forecasted hly load.

\subsubsection{Must Run and off Constraint}

Generators are required to provide energy or to shutdown due to some operational circumstances:

$$
\begin{aligned}
& I_{g t}=1,\left(t_{1} \leq t \leq t_{2}\right)(g=1, \ldots, M R), \\
& I_{g t}=0 .\left(t_{1} \leq t \leq t_{2}\right)(g=1, \ldots, M O) .
\end{aligned}
$$

The $M R$ and $M O$ indicates the $g$ th unit that must run and off within the time interval, respectively.

\subsection{Objective Function for Outage Operation}

During an outage event, the objective is to supply the energy to critical loads from standby generators in economically efficient manner. The objective function for the outage operation $C_{I}$ is similar to Equation (1), but is intended only for contingency purposes:

$$
\text { Minimize: } \quad C_{I}(t)=\sum_{x=1}^{N_{x}}\left[F_{x}\left(P_{G, x t}^{c} I_{x t}\right) N 1_{G, x t}^{c}+S U_{x}\right]+S D_{x}+\sum_{z=1}^{N_{z}}\left[C_{t} P_{S, z t}^{c} N 1_{S, z t}^{c}\right]+C_{t} P_{M, t}^{c} N 1_{M, t}^{c} .
$$

The index $i$ refers to the generating units in $N-1$ state and $N_{x}$ and $N_{z}$ is the total thermal generating units and ESS units in $N-1$ state: $N 1_{G, x t}^{c}$ refers the $N-1$ contingency state of unit $x$ at time $t . N 1_{S, z t}^{c}$ is the $N-1$ state of ESS.

The operational control constraints are applied using Equations (2) and (4)-(11). A N-1 contingency criterion constraint is considered during an outage event. The $\mathrm{N}-1$ system compliance ensures the grid can survive any single outage in any links, and also the line across the MV/LV level grid. The generation and the transmission line output are constrained in the contingency situation:

$$
\begin{aligned}
& P_{G, x}^{\min } I_{x t} N 1_{G, x t}^{c} \leq P_{G, x t}^{c} \leq P_{G, x}^{\max } I_{x t} N 1_{G, x t}^{c}, \\
& \left|P_{M, t}^{c}\right| \leq P_{M}^{\max } N 1_{M, t}^{c} .
\end{aligned}
$$

The superscript $c$ denotes the contingency state. The $N 1_{G, x t}^{c}$ in Equation (13) denotes the binary parameter for $N-1$ contingency state of $x$ at time $t$. The $N 1_{G, x t}^{c}=0$ when the generator is in contingency state and $N 1_{G, x t}^{c}=1$ for normal operations. If the $x$ th unit is not committable $\left(I_{x t}=0\right)$, or the unit is in outage with $N 1_{G, x t}^{c}=0$, the $x$ th unit generation output is zero. This is to indicate that an offline generator cannot respond during the contingency situation. Hence, the committable generating units are only allowed to operate in the contingency state. The operating constraints for committable generators during the contingency state will remain the same.

Similarly, $N 1_{M, t}^{c}$ in Equation (14) denotes the binary parameter for $N-1$ contingency state of the transmission line between the MV and LV level. The $N 1_{M, t}^{c}=0$ forces the transmission flows to be zero when the transmission line is in contingency state and otherwise, $N 1_{M, t}^{c}=1$ for normal operations. Depending on the scale of damages, for example, if the transmission line is not affected, i.e., $N 1_{M, t}^{c}=1$, the affected $x$ th generators in a node $\left(N 1_{G, x t}^{c}=0\right)$ will be in islanded mode of operation, whereas the unaffected area will be in normal operation mode. 
The power balance equation in the contingency state is:

$$
\sum_{x=1}^{N_{x}} P_{G, x t}^{c}+P_{M, t}^{c}+\sum_{y=1}^{N_{y}} P_{R, y t}^{c}+\sum_{z=1}^{N_{z}} P_{S, z t}^{c}=P_{D, t}^{c} .
$$

In order to continuously supply energy to critical infrastructure during the outage operation, the functionality of ESS is critical and therefore is modelled in the grid architecture.

\subsection{ESS Model}

ESS stores energy at times of low energy market prices (off-peak electricity demand), discharges the stored energy during high electricity market prices, low grid generation, and also the contingency situation in compensating the generation shortages. Methods of ESS operation and modelling by Bahramirad [25], Howlader et al. [31] are followed.

There are three important operating modes in ESS, which are the charging, discharging, and idle mode. For the contingency situation, the energy storage will discharge, based on the available storage capacity.

The energy stored in ESS is the state of charge (SOC). The SOC for ESS is formulated as:

$$
P_{S O C, s t}=P_{S O C, s(t-1)}-P_{S, s t} \Delta t .
$$

$P_{S O C, s t}$ is the SOC of ESS, $\Delta t$ is the time interval in the SOC, and $s$ indexes ESS unit. The $P_{S, s t}$ in this case is denoted with positive and negative magnitude depending on the charge and discharge mode. From Equation (16), during the charging mode, ESS is storing the energy, the $P_{S, s t}$ is negative and thus the value of $P_{S O C, s t}$ increases. On the contrary, during the discharging mode, ESS is said in generating the energy and the $P_{S, s t}$ is positive. Then, the value of $P_{S O C, s t}$ decreases. As clarified by Howlader et al. [31], depending on the current storage technology, ESS is with the operating margin capacity of $80 \%$ upper limit and $20 \%$ lower limit:

$$
P_{S O C, S}^{\min }[=20 \%] \leq P_{S O C, s t} \leq P_{S O C, S}^{\max }[=80 \%] .
$$

In this case, it is assumed that the ESS is allowed to discharge the energy until ESS is partially exhausted (20\%), and charge at $80 \%$ of the maximum capacity. Additionally, the instant charging/discharging capability is assumed as soon as a signal is sent by the microgrid controller. The charging and discharging mode of ESS is remained the same during a contingency event. However, the proportion of SOC may change and Equations (16) and (17) is modified as:

$$
\begin{aligned}
& P_{S O C, z t}^{c}=P_{S O C, z(t-1)}^{c}-P_{S, z t}^{c} \Delta t, \\
& P_{S O C, z}^{\min }[=20 \%] \leq P_{S O C, z t}^{c} \leq P_{S O C, z}^{\max }[=80 \%] .
\end{aligned}
$$

The operation of ESS is accomplished by adding the generation output to the energy balance equation (normal and outage operation) in Equations (3) and (15).

\subsection{Resilience Metric}

In order to access the resilience of a grid, a performance metric indicator is established. As in line with Cano-Andrade et al. [18] and Bollinger [19], the resilience metric in this paper is represented by the extent in which the amount of energy demand within consumers are met when there is a disturbance in the grid. The performance metric to calculate the resiliency is based on the fraction of demand served, or the amount of the energy produced by the microgrid during an outage $[18,19]$. 
The resilience coefficient in this case is therefore the fraction of demand served at $d$ th consumer $\left(P_{D, d t}^{c}\right)$ divided by the overall demand $\left(P_{D, t}^{c}\right)$ in the contingency state:

$$
\alpha_{R}=\sum_{t=1}^{N_{t}} \frac{P_{D, d t}^{c}}{P_{D, t}^{c}+P_{D, d t}^{c}} .
$$

\section{Simulation Modelling and Settings}

An IEEE 14-node test topology is used as the benchmark grid in this case. In order to perform a microgrid simulation in a IEEE test system, the original capacity of $280 \mathrm{MW}$ in the test grid has been scaled down, such that each of the nodes can be configured as microgrid with the total $6.70 \mathrm{MW}$ of the load capacity $(\leq 10 \mathrm{MW})$. A random configuration of buildings consisting of eight blocks of offices, one large hospital, two outpatient clinics, three supermarkets, three warehouses blocks and 1000 residential houses has been simulated for a duration of one day. Samples of demand profiles are obtained from the portals (Elexon [36], OpenEI [37]). Outages of different durations are simulated (as illustrated in different grid operations). Contingency tests (Ejebe and Wollenberg [38]) are performed by simulating single line failure in each case. The overall distribution of the generation and consumer loads in the grid architecture is modelled using the microgrid configuration in Figure 1 and Table 1. The distribution of load profile in this case is not intended to include the profiles of commercial services and domestic households within the same node. This is because most commercial services are connected with their own substations due to the huge amount of loads required.

Two types of RES - wind and solar-powered PV for LV and MV with 0.5 MW generating capacity of each are considered in this example, where the characteristic and generation profiles of RES are adopted from Jayaweera and Islam [39] and Elexon [40]. The generation data from Elexon [40] is further rescaled to fit the current microgrid capacity. Four types of thermal units for microgrid which are non-renewable backup generators are considered in this example with 0.5-1.0 MW capacities, alongside with two mid-scale thermal (2.0 MW capacity each) for MV level grid. Specification of thermal units were originally adopted from Bahramirad [25], Lau et al. [27], with implemented modifications to fit the required generations to the load demands.

Two identical ESS units with a total of $2 \mathrm{MW}$ storage capacity (1 MW each) are included in the simulation. Each ESS can be fully charged within $6 \mathrm{~h}$ in order to reach the maximum SOC of $9.6 \mathrm{MWh}$ (within $20-80 \%$ of capacity).

The demand from each node is summed in order to provide the total aggregated demand across the grid $\left(P_{D, t}\right)$. The timeline for the simulation is limited to $24 \mathrm{~h}$ with hly-based temporal resolution. As the time interval for the optimization is $1 \mathrm{~h}, \Delta t=1$ is applied in Equations (16) and (18).

The wholesale electricity market price data is obtained from the EPEX SPOT portal [41,42]. It is one of the UK's power exchange providing the trading and clearing services for the wholesale market. EPEX SPOT portal provides historical data of the reference price data for the electricity. In this case, the 2017 year of reference price data is adopted, with additional calculation to compute the $24 \mathrm{~h}$ average whole electricity price data.

The optimization problems are solved using the MATLAB ${ }^{\circledR}$ R2017b platform (version 9.3.0.713579, 64-bit, the MathWorks Inc., Natick, MA, USA) with 7th Generation Intel ${ }^{\circledR}$ Core i5-7267U (3.1 GHz dual-core, $4 \mathrm{MB}$ cache, up to $3.5 \mathrm{GHz}$ ) CPU and $8 \mathrm{~GB}$ (2133 MHz LPDDR3) of RAM. The simulations for optimization problems along with graphical illustration result plots are obtained for the duration of $0.8 \mathrm{~s}$, thereby requiring minimal amount of computational efforts. The dual-simplex algorithm is applied for the LP problem, where the solution starts better than optimum but remains infeasible until the true optimum is reached along with feasible solution [43]. The conventional operation of the grid is simulated with economic-based islanding in responding to the market price. 
Table 1. Number of standby generator, energy storage system (ESS), types of consumer profiles and their loads included in the low voltage (LV) network.

\begin{tabular}{ccccc}
\hline \multirow{2}{*}{ Node No. } & \multicolumn{2}{c}{ Number of Standby Generator } & Number of ESS & Consumer Profiles \\
\cline { 2 - 3 } & Non-Renewable & Renewable & & \\
\hline 1 & 2 & 2 & 2 & Household \\
2 & 2 & 2 & 2 & Office \\
3 & 4 & 1 & 4 & Hospital \\
4 & 2 & 1 & 2 & Outpatient clinic \\
5 & 2 & 1 & 2 & Warehouse \\
6 & 2 & 1 & 2 & Supermarket \\
\hline
\end{tabular}

\section{Results and Analyses}

Three different cases (normal grid operation, the outage operation and complete grid outage) are implemented into the model to observe changes in the grid operation cost and the resilience metric. It is not the scope of this paper to generalize the evaluation of established cases. This is because different cases would have different projected outage timelines, and requirement purposes.

\subsection{Case 1-Normal Grid Operation}

In the first scenario, there is no outage and hence the normal operation mode is applied. The top panel of Figure 3 shows the resultant energy generation distribution and its balanced/optimized generation for normal operation. The legends 'Main grid generation', 'Nominal generation' and 'Balanced generation' in Figure 3 denote the generation from mid or high level grid, the expected generation in responding to the total demand $\left(P_{D, t}\right)$ without islanding capability, and optimized generation dispatches with the islanding capability from the standby generator and ESS in the microgrid, respectively. When the electricity price is low at h $0-17)$, the grid is in normal operation where the energy is actually imported from the main grid to LV grid. At times of high electricity prices, the standby generator and ESS in LV level are triggered by the microgrid controller, along with the available RES to generate energy in order to satisfy the current load. At these instances, the microgrid is in islanded mode of operation and there is no energy purchase from the main grid. After that, when the electricity price is low (at h 21-24), the microgrid reconnects back to the grid as soon as the electricity market price is at the acceptable threshold value. As it is not an outage operation, the resilience coefficient in this case is zero.

The cost of operation for the whole grid solution is shown in the top panel of Figure 3. In this case, the conventional is defined as when only grid-connected mode of operation is allowed. In contrast, the optimized grid solution is when there is an islanded operation. The overall cost saving in this $24 \mathrm{~h}$ of simulation runs is $£ 291.56$. Hence, the first scenario shows that the positive aspects of the grid avoid drawing the electricity from the main grid at times of high electricity prices. The cost saving calculated in this case, however, excludes the other factors such as the maintenance and other overhead costs from RES, standby generator and ESS. 

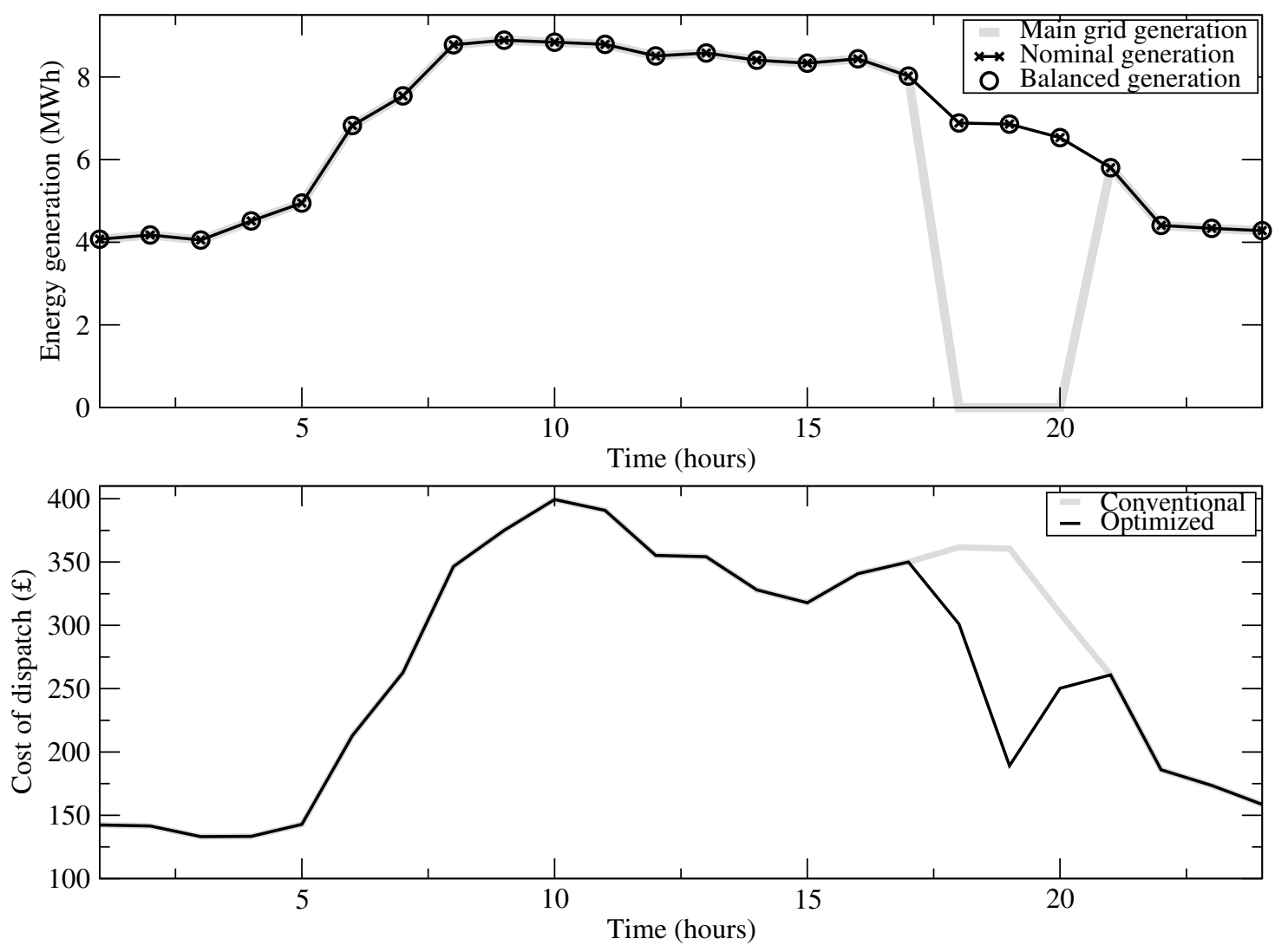

Figure 3. Top panel: resultant energy generation distribution and its balanced/optimized generation for normal operation; bottom panel: cost of operation between the conventional and optimized solution.

\subsection{Case 2-The Outage Operation}

In this case, an earthquake is assumed that damages one of the electricity connections. The line connecting to the large hospital load (Node 3) and the main grid is cut off (CB5 is opened in Figure 1) and therefore is in the contingency state. The hospital load is therefore isolated from the grid. The outage occurs at $\mathrm{h} 900$ for the duration of six hours. The load will be recovered through the committable on-site standby generator, RES and ESS. The remaining loads, similar to Case 1 , is in the normal operation mode, but the economic-islanding operation feature is disabled (as the outage event is the major issue to be addressed).

The distribution of energy generation following the outage is shown in Figure 4. The legends 'Nominal generation', 'Hospital load', and 'Balanced hospital load' in Figure 4 are the expected generation in responding to the overall demand $\left(P_{D, t}^{c}\right)$ during the islanding operation, the expected hospital demand, and the balanced/optimized supply amount for the hospital load during the outage. The huge drop in load between $\mathrm{h} 900-1500$ in the main grid is due to the outage load in the hospital where the hospital load is isolated from the main grid. During the outage period, the hospital load is compensated by the available diesel-powered standby generators, ESS and RES. The amount of generation required for the hospital load is secured during the outage. 


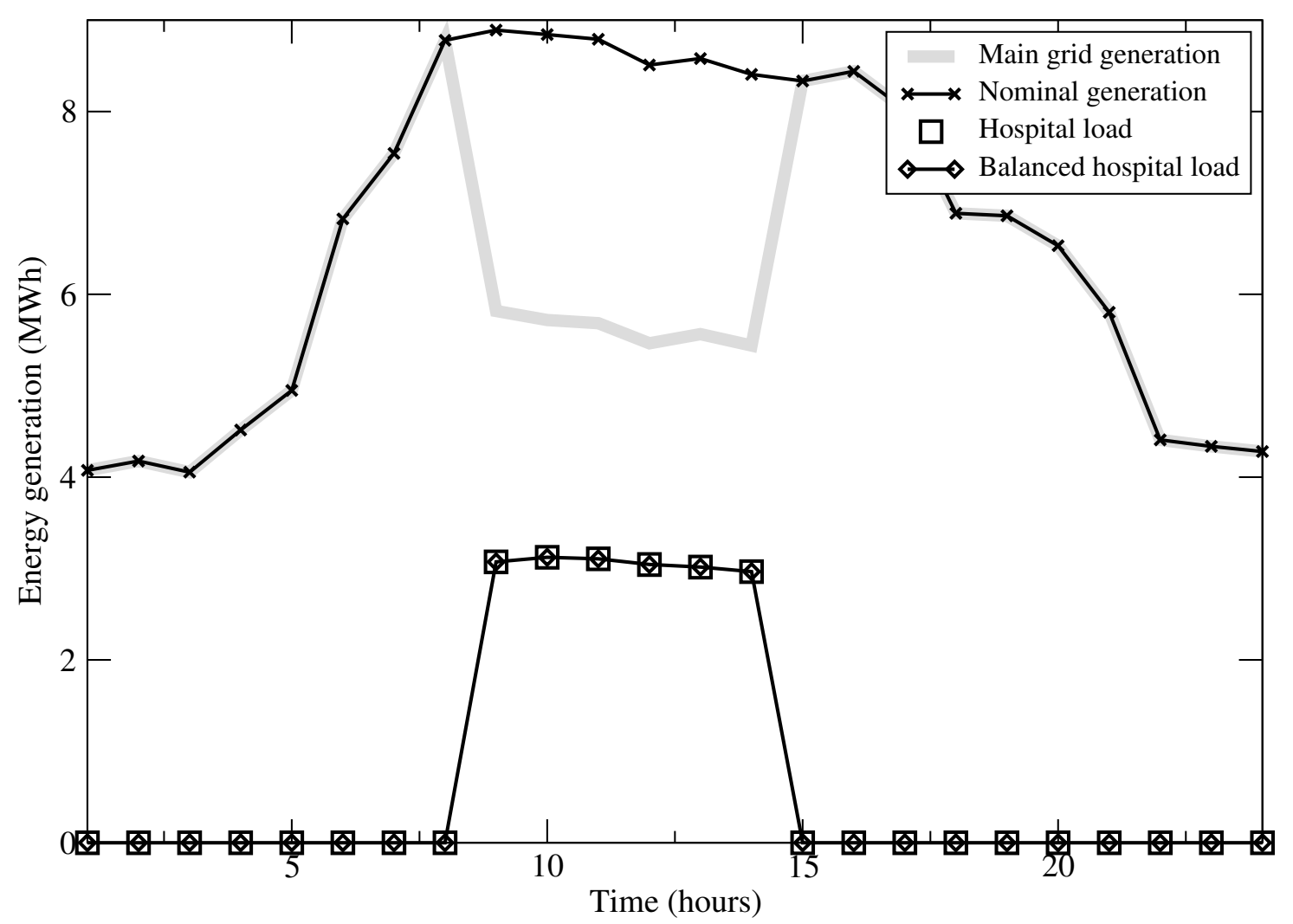

Figure 4. The resultant energy generation distribution, the nominal generation and the generation distribution of hospital load during an outage.

The SOC of ESS is shown in the top panel of Figure 5. It is assumed that the ESS is in full capacity ESS is in 'idle' state awaiting the dispatching instruction. At the time of outage period (at h 900-1500), ESS generates (discharging) energy to the load in order to compensate the imbalances of demand shortage in Node 3. At h 1500, as the outage problem is solved, ESS is allowed to charge subject to low peak electricity price (from h 1500-1700 and from 2100 onwards) in the preparation for the next stage of scheduling horizon. During h 1700-2100, ESS is in idle mode due to high peak electricity market price, thus ESS is prevented from drawing electricity from the main grid. Additionally, as the outage has only been resolved for a short duration, henceforth the economic-islanding is not possible in this case due to security-of-supply recovery requirements. Furthermore, ESS capacity is not required to be fully depleted to allow for charging, instead the charging mode can be triggered as soon as the cheap electricity price occurs.

The $N-1$ criterion is applied where a single electricity connection line connecting the main grid to the hospital load is in outage. The time-dependent resilience distribution of the system is shown in the bottom panel of Figure 5. It can be seen that the resilience during the outage is average-high. The zero coefficient of resilience indicates the normal state of grid operation. The overall resiliency of the system is calculated as 0.352 using Equation (20). Such calculation demonstrates the capability and resiliency of the grid to withstand the outage in an efficient manner. In contrast, if there is a huge amount of loads deviated during the outage, and the resultant resilience coefficient is low, this indicates the low resiliency of the grid in sustaining the required loads.

The cost optimized in this case is $£ 187.77$. This would be the optimal way of dispatching generating units during the outage, while preserving the critical loads. However, such cost saving should not to be related to the economical operation of dispatchable units during off-peak periods, as any other forms of environmental concerns such as emissions from air particles should be noted. 

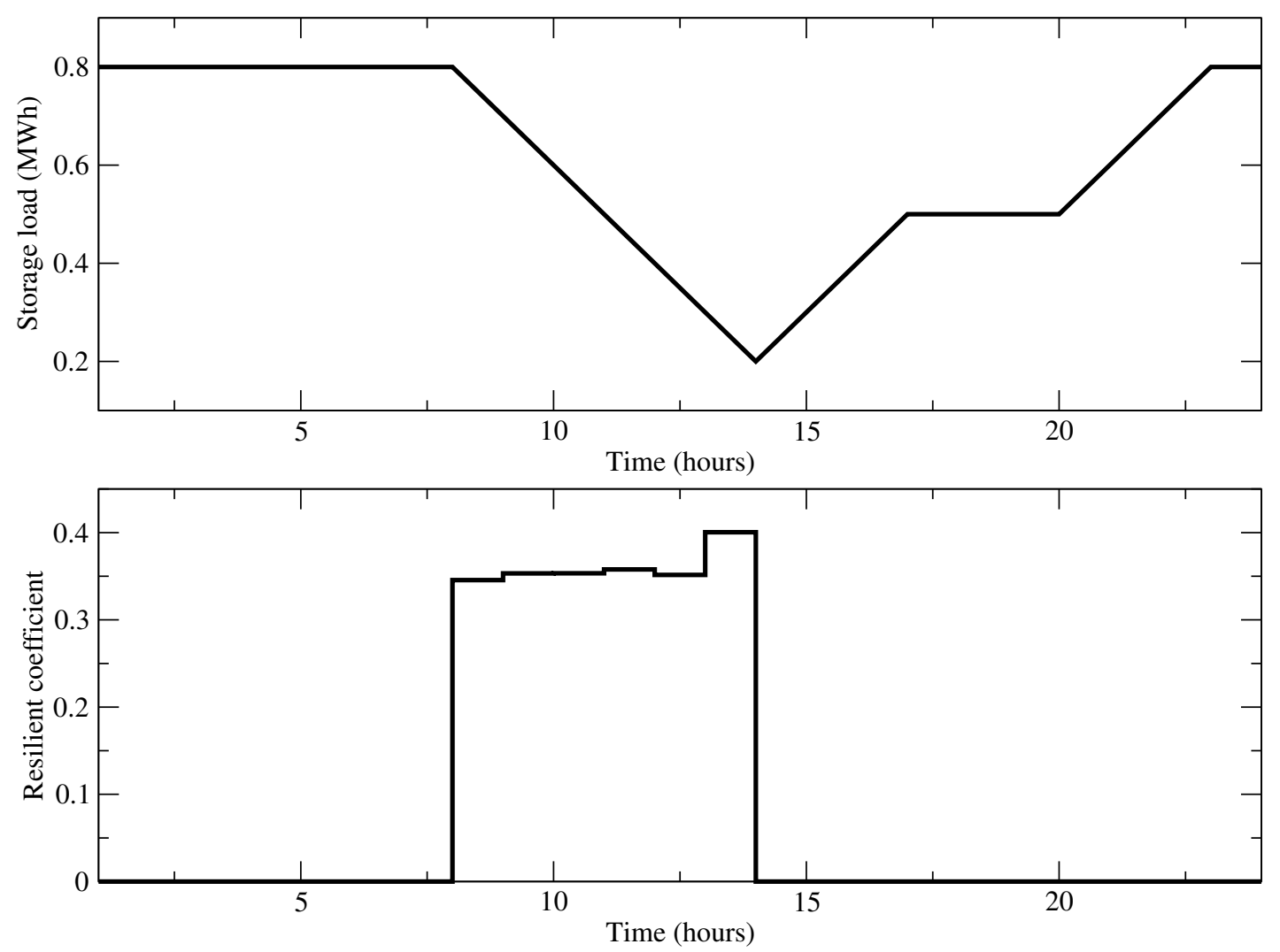

Figure 5. Top panel: the state of charge (SOC) in energy storage system (ESS) during an outage; bottom panel: the resilience distribution of the system following an outage.

\subsection{Case 3-Complete Grid Outage}

The third case accounts the same disaster scenario from the second case, but with the complete outage of the electricity connection between the high-mid and the low level grid (CB2 is opened referring to Figure 1). Consequently, the entire low level grid is isolated from the rest of the grid and the islanding capability is triggered. The outage period and duration are remained the same (at $\mathrm{h}$ 900-1500). In addition, the economic-islanding operation feature is disabled.

The energy generation distribution and the balanced generation for the low level grid is shown in the top panel of Figure 6. It is notable that, due to the complete grid outage, the overall main grid load drops to zero at $h$ 900-1500. Therefore, the islanded mode operates on the LV level during the outage periods. The LV level loads are successfully balanced through the optimized dispatching of generating units. As soon as the outage is solved, the islanded operation terminates and instantaneous main grid re-connection is achieved that permits the normal operation mode.

The cost of operation for the conventional and optimized grid solution for the third scenario is presented in the bottom panel Figure 6. The optimized cost for dispatching of generating units is $£ 242.80$. Additionally, the overall resilience coefficient of this case is 1 , where all demands are met without failing. However, it can be noted that at some instance the dispatching of optimized units are more costly than the normal operation to compensate for the demand during the outage. This shows the trade-off in between the cost and grid resilience, as the main priority is to ensure that the security of energy demand is met entirely during the outage to prevent complete blackouts. Therefore, the optimization module aims at optimizing the operation cost but may incur additional costs at some time instance due to requirements in adhering to grid operational security constraints (i.e., more expensive fast acting generators are required to operate). Such demonstration illustrates the capability of the microgrid to withstand the complete outage from the main level grid while maintaining the grid resilience. 

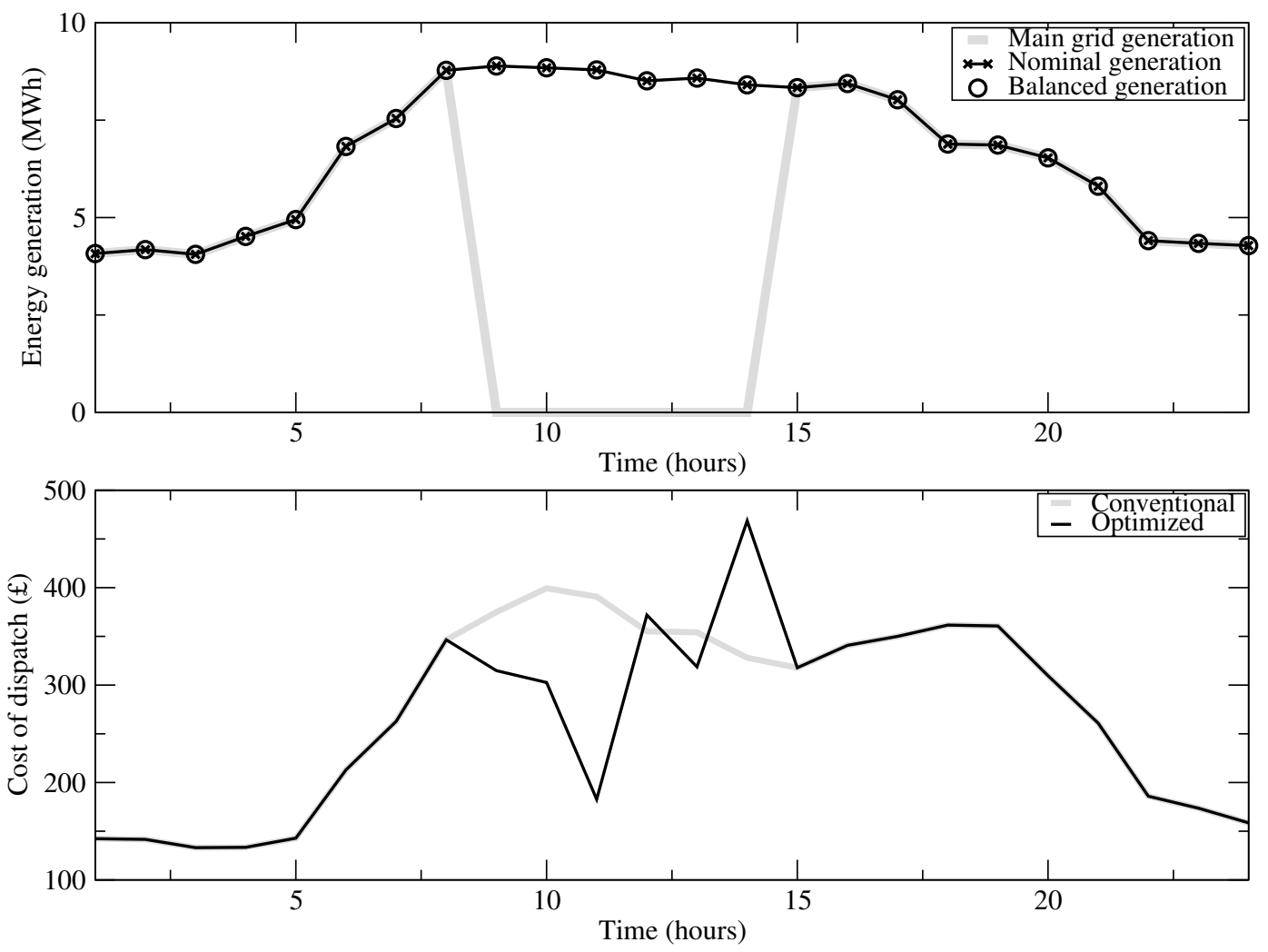

Figure 6. Top panel: resultant energy generation distribution and its balanced/optimized generation during complete grid outage; bottom panel: cost of operation between the conventional and optimized solution during the complete grid outage.

\section{Conclusions}

This paper proposes a resilient-based optimization module to balance the monetary costs and resilience. The outage with $N-1$ contingency criterion is applied by isolating the grid portions from the main load, depending on the scale of outages. The proposed model allows for the whole integration of the main grid supply, electricity connections, DER such as standby generators, ESS, RES, energy demand profiles and simulation of grid outages. The major breakthrough of this paper is the capability of performing the time-dependent resilience analysis of microgrid operation. The established resilience-optimization model represents a smart grid network that maintains the overall resilience across the grid subject to adverse events.

The involvement of ESS as well as RES in different scenarios is not only capable of providing contingency response but also has successfully mitigated the amount of generations produced by polluting standby generators, thus improving the environmental health.

The developed optimization model acts as the fundamental approach that can further support larger urban electricity grids with high flexibility. However, precautionary measures of microgrid penetrations should be performed in the future work to determine the marginal level of microgrid penetrations that will guarantee the grid safety and superior operations. As the current model concentrating on single line failure, a meshed grid topology for the model is essential not to only examine a single failure, but also to examine the multiple cascading failures across the grid. Future work should extend the capability of the proposed method. Additionally, as it is not the scope of this paper to compare different grid configurations, future work should consider the variety of grid configurations (for instance, baseline and the decarbonisation scenario) for performance evaluation purposes.

Author Contributions: E.T.L. and K.K.C. conceived and designed the experiments. E.T.L. performed the experiments. E.T.L. and K.K.C. coordinated the research that led to the results presented in this paper. E.T.L. wrote the manuscript. K.K.C., Y.C. and J.L. supported the modification and improvement of this paper. All authors 
contributed state-of-the-art journal materials and analysis modules. All authors contributed in structuring the study.

Funding: This work was fully supported by the Joint Program Initiative (JPI) Urban Europe via the IRENE project. Grant Reference: ES/M008509/1.

Conflicts of Interest: The authors declare no conflict of interest.

\section{Nomenclature}

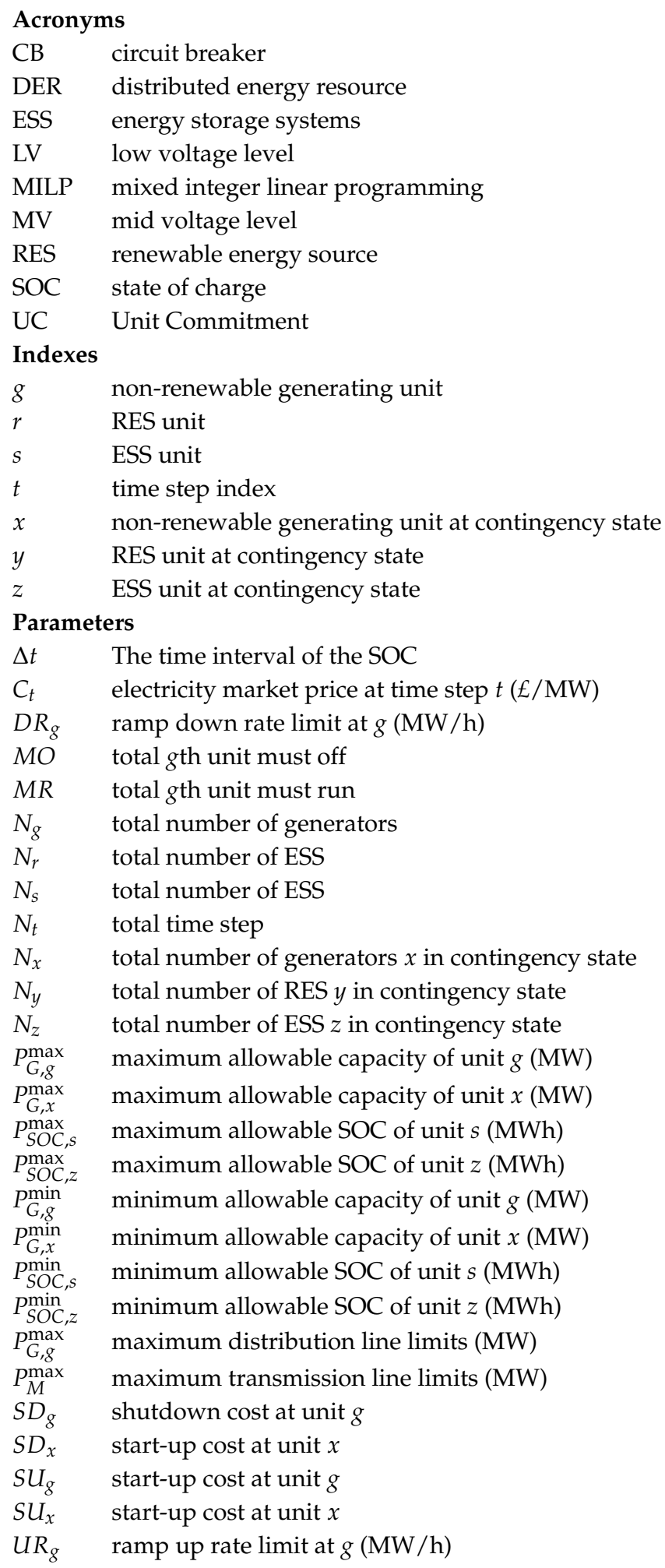




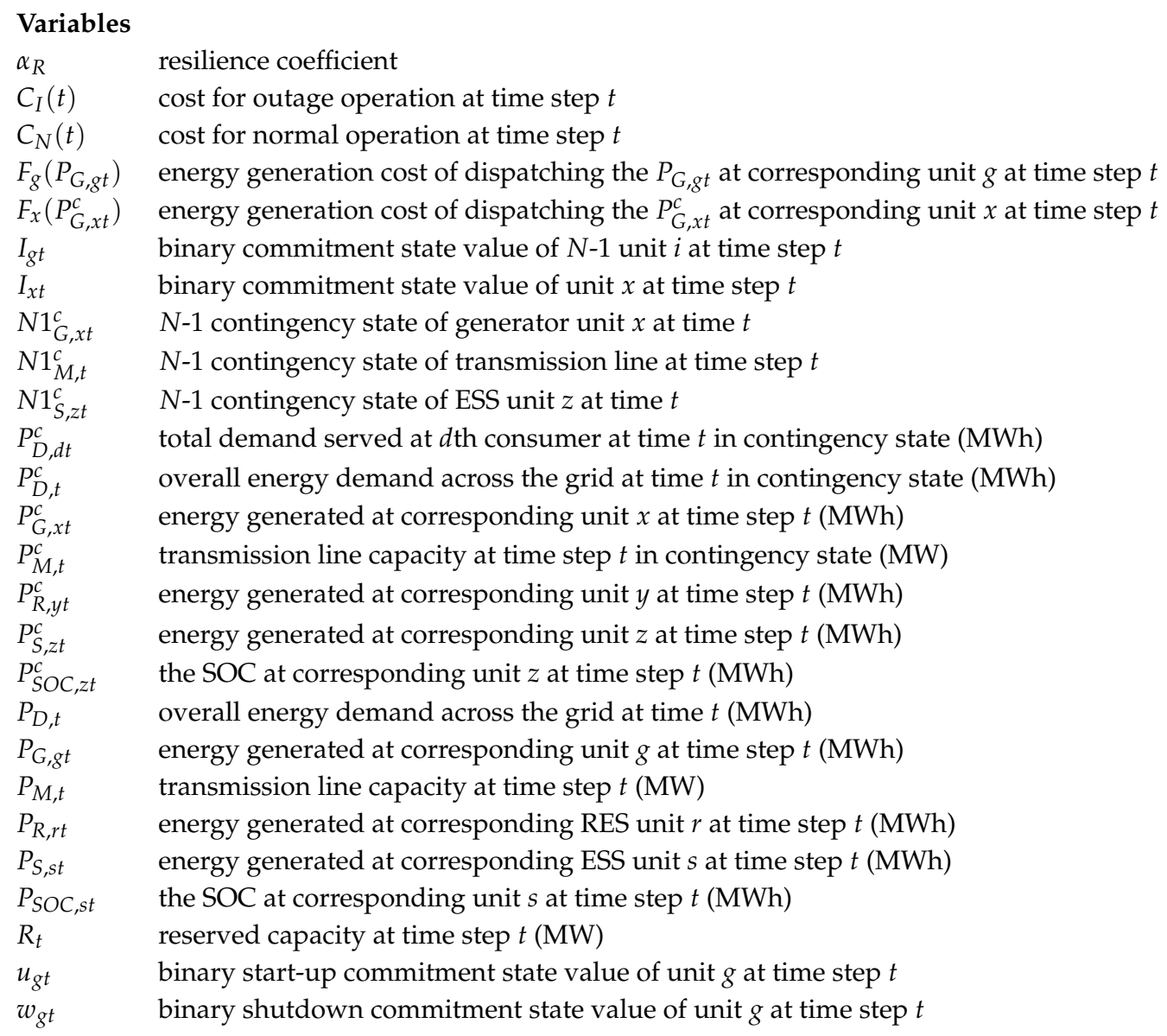

\section{References}

1. Erol-Kantarci, M.; Kantarci, B.; Mouftah, H.T. Reliable overlay topology design for the smart microgrid network. IEEE Netw. 2011, 25, 38-43. [CrossRef]

2. Alam, S.; Sohail, M.F.; Ghauri, S.A.; Qureshi, I.M.; Aqdas, N. Cognitive radio based smart grid communication network. Renew. Sustain. Energy Rev. 2017, 72, 535-548. [CrossRef]

3. Chaouachi, A.; Kamel, R.M.; Andoulsi, R.; Nagasaka, K. Multiobjective intelligent energy management for a microgrid. IEEE Trans. Ind. Electron. 2013, 60, 1688-1699. [CrossRef]

4. Vaccaro, A.; Popov, M.; Villacci, D.; Terzija, V. An integrated framework for smart microgrids modeling, monitoring, control, communication, and verification. IEEE Proc. 2011, 99, 119-132. [CrossRef]

5. Olivares, D.E.; Mehrizi-Sani, A.; Etemadi, A.H.; Cañizares, C.A.; Iravani, R.; Kazerani, M.; Hajimiragha, A.H.; Gomis-Bellmunt, O.; Saeedifard, M.; Palma-Behnke, R.; et al. Trends in microgrid control. IEEE Trans. Smart Grid 2014, 5, 1905-1919. [CrossRef]

6. Hatziargyriou, N.; Asano, H.; Iravani, R.; Marnay, C. Microgrids. IEEE Power Energy Mag. 2007, 5, 78-94. [CrossRef]

7. Winkler, J.; Dueñas-Osorio, L.; Stein, R.; Subramanian, D. Performance assessment of topologically diverse power systems subjected to hurricane events. Reliab. Eng. Syst. Saf. 2010, 95, 323-336. [CrossRef]

8. Elsied, M.; Oukaour, A.; Gualous, H.; Brutto, O.A.L. Optimal economic and environment operation of micro-grid power systems. Energy Convers. Manag. 2016, 122, 182-194. [CrossRef]

9. Arefifar, S.A.; Ordonez, M.; Mohamed, Y.A.I. Energy management in multi-microgrid systems-Development and assessment. IEEE Trans. Power Syst. 2017, 32, 910-922.

10. Shi, W.; Xie, X.; Chu, C.C.; Gadh, R. Distributed optimal energy management in microgrids. IEEE Trans. Smart Grid 2015, 6, 1137-1146. [CrossRef]

11. Parisio, A.; Rikos, E.; Tzamalis, G.; Glielmo, L. Use of model predictive control for experimental microgrid optimization. Appl. Energy 2014, 15, 37-46. [CrossRef] 
12. Hossienzadeh, M.; Salmasi, F.R. Robust optimal power management aystem for a hybrid AC/DC micro-grid. IEEE Trans. Sustain. Energy 2015, 6, 675-687. [CrossRef]

13. Chen, C.; Duan, S.; Cai, T.; Liu, B.; Hu, G. Smart energy management system for optimal microgrid economic operation. IET Renew. Power Gener. 2011, 5, 258-267. [CrossRef]

14. Yang, T.; Zhang, Y.; Wang, Z.; Pen, H. Secondary frequency stochastic optimal control in Independent Microgrids with VSG-controlled energy storage systems. Energies 2018, 11, 2388. [CrossRef]

15. Wang, S.; Li, L.; Huang, B.; Jiang, L. Hierarchical and decentralized optimization method based on multiagent system for multiple AC/DC hybrid microgrids. J. Renew. Sustain. Energy 2018, 10, 045302. [CrossRef]

16. Chettibi, N.; Mellit, A.; Sulligoi, G.; Pavan, A.M. Adaptive neural network-based control of a hybrid AC/DC microgrid. IEEE Trans. Smart Grid 2018, 9, 1667-1679. [CrossRef]

17. Panteli, M.; Pierluigi, M. The grid: Stronger, bigger, smarter? IEEE Power Energy Mag. 2015, 13, 58-66. [CrossRef]

18. Cano-Andrade, S.; Spakovsky, M.R.; Fuentes, A. Multi-objective optimization for the sustainable-resilient synthesis/design/operation of a power network coupled to distributed power producerrs via microgrids. In Proceedings of the ASME 2012 International Mechanical Engineering Congress \& Exposition (IMECE 2012), Houston, TX, USA, 9-15 November 2012; pp. 1-16. [CrossRef]

19. Bollinger, L.A. Fostering Climate Resilient Electricity Infrastructure. 2015. Available online: http:/ / repository. tudelft.nl/islandora/object/uuid:d45aea59-a449-46ad-ace1-3254529c05f4/datastream/OBJ/download (accessed on 6 December 2016).

20. Khodaei, A. Resiliency-oriented microgrid optimal scheduling. IEEE Trans. Smart Grid 2014, 5, $1584-1591$. [CrossRef]

21. Liu, X.; Shahidehpour, M.; Li, Z.; Liu, X.; Cao, Y.; Bie, Z. Microgrids for enchancing the power grid resilience in extreme conditions. IEEE Trans. Smart Grid 2017, 8, 589-597. [CrossRef]

22. Romero, N.R.; Nozick, L.K.; Dobson, I.D.; Xu, N.; Jones, D.A. Transmission and generation expansion to mitigate seismic risk. IEEE Trans. Power Syst. 2013, 28, 3692-3701. [CrossRef]

23. Ouyang, M.; Dueñas-Osorio, L. Time-dependent resilience assessment and improvement of urban infrastructure systems. Chaos 2012, 22, 320-330. [CrossRef] [PubMed]

24. Detroja, K.P. Optimal autonomous microgrid operation: A holistic view. Appl. Energy 2016, 173, $320-330$. [CrossRef]

25. Bahramirad, S. Economic and Emergency Operations of the Storage System in a Microgrid; Degree Project; School of Electrical Engineering, KTH Royal Institute of Technology: Stockholm, Sweden, 2012.

26. Liang, H.; Zhuang, W. Stochastic modelling and optimization in a microgrid: A survey. Energies 2014, 7, 2027-2050. [CrossRef]

27. Lau, E.T.; Yang, Q.; Taylor, G.A.; Forbes, A.B.; Wright, P.S.; Livina, V.N. Optimisation of costs and carbon savings in relation to the economic dispatch problem as associated with power system operation. Electr. Power Syst. Res. 2016, 140, 173-183. [CrossRef]

28. Zendehdel, N.; Karimpour, A.; Oloomi, M. Optimal unit commitment using equivalent linear minimum up and down time constraints? In Proceedings of the 2nd IEEE International Conferencec on Power and Energy (PECon 08), Johor Bahru, Malaysia, 1-3 December 2008; pp. 1021-1026. [CrossRef]

29. Hedman, K.W.; Ferris, M.C.; O’Neill, R.P.; Fisher, E.B.; Oren, S.S. Co-Optimization of generation unit commitment and transmission switching with N-1 reliability. IEEE Trans. Power Syst. 2010, 25, 1052-1063. [CrossRef]

30. Khodaei, A. Microgrid optimal scheduling with multi-period islanding constraints. IEEE Trans. Power Syst. 2014, 29, 1383-1392. [CrossRef]

31. Howlader, H.O.R.; Matayoshi, H.; Senjyu, T. Distributed generation integrated with thermal unit commitment considering demand response for energy storage optimization of smart grid. Renew. Energy 2016, 99, 107-117. [CrossRef]

32. Chiandussi, G.; Codegone, M.; Ferrero, S.; Varesio, F.E. Comparison of multi-objective optimization methodologies for engineering applications. Comput. Math. Appl. 2012, 63, 912-942. [CrossRef]

33. Edurite. Advantages and Disadvantages of Linear Programming. 2015. Available online: http://www. edurite.com/kbase/advantages-and-disadvantages-of-linear-programming (accessed on 30 January 2017).

34. Weron, R. Electricity price forecasting: A review of the state-of-the-art with a look into the future. Int. J. Forecast. 2014, 30, 1030-1081. [CrossRef] 
35. Pezzutto, S.; Grilli, G.; Zambotti, S.; Dunjic, S. Forecasting electricity market price for end users in EU 28 until 2020-Main factors of influence. Energies 2018, 11, 1460. [CrossRef]

36. Elexon. What Is Load Profile? 2016. Available online: https://www.elexon.co.uk/reference/technicaloperations/profiling/ (accessed on 6 December 2016).

37. OpenEI. Commercial and Residential Hourly Load Profiles for All TMY3 Locations in the United States. 2016. Available online: http://en.openei.org/datasets/dataset/commercial-and-residential-hourly-loadprofiles-for-all-tmy3-locations -in-the-united-states (accessed on 6 December 2016).

38. Ejebe, G.C.X.; Wollenberg, B.F. Automatic contingency selection. IEEE Trans. Power Appl. Syst. 1979, PAS-98, 97-109. doi:10.1109/TPAS.1979.319518. [CrossRef]

39. Jayaweera, D.; Islam, S. Security of supply in active distribution networks with PHEV-based strategic microgrids. Parts Ser. Reliab. Sustain. Electr. Power Energy Syst. Manag. 2014, 17-31. [CrossRef]

40. Elexon. Elexon Portal. Elexon Ltd. 2017. Available online: https://www.elexonportal.co.uk/news/latest? cachebust=migsr3hufu (accessed on 3 December 2018).

41. EPEX SPOT. Latest Market Results. 2018. Available online: https://www.apxgroup.com (accessed on 15 November 2018).

42. EPEX SPOT. UKPX Auction Historical Data. 2018 Available online: https://www.apxgroup.com/marketresults/apx-power-uk/ukpx-auction-historical-data/ (accessed on 15 November 2018).

43. Gupta, P.K.; Hira, D.S. Operations Research, revised ed.; S Chand \& Co Ltd.: New Delhi, India, 2008.

(c) 2018 by the authors. Licensee MDPI, Basel, Switzerland. This article is an open access article distributed under the terms and conditions of the Creative Commons Attribution (CC BY) license (http://creativecommons.org/licenses/by/4.0/). 